Nuclear Physics B (Proc. Suppl.), 2001, v. 102\&103, pp. 311-315.

\title{
Affine Root Systems and Dual Numbers
}

\author{
I.V. Kostyakov, N.A. Gromov and V.V. Kuratov
}

Department of Mathematics, Syktyvkar Branch of IMM UrD RAS, Kommunisticheskaya st., 24, Syktyvkar, 167000, Russia

The root systems in Carroll spaces with degenerate metric are defined. It is shown that their Cartan matrices and reflection groups are affine. Due to the geometric consideration the root system structure of affine algebras is determined by a sufficiently simple algorithm.

It is known that nonsemisimple Lie groups and algebras may be obtained by introducing degenerate (nilpotent) directions into the main notions of semisimple Lie groups and algebras theory [1]. It is an algebraic approach to the limit passage from simple to nonsemisimple groups suggested by E.Inönü and E.P.Wigner [2]. Further kind of algebraic contractions is graded contractions [3].

All models of modern physics appear due to underlying symmetries. Simple finite Lie algebras and infinite affine Kac-Moody algebras play an important role in describing such symmetries. Interest in models with nonsemisimple symmetry is growing $[4,5]$ and these models may be investigated with the help of contractions. There are some models produced by contractions from some initial ones, for example usual Toda chains may be obtained from affine Toda chains by the contractions from infinite-dimensional affine Lie algebra to finitedimensional simple Lie algebra [6,7]. Properties of models with different Lie algebras may be related if there are relations between their algebras. In this paper the relations between root systems of finite and infinite-dimensional algebras are considered. These relations are determined by a geometric way.

It is not well known $[1,9,10]$, that all $3^{n} n$-dimensional spaces of constant curvature may be obtained by multiplications of orthonormal basis elements of Euclidean space by certain products of the parameters $j_{k}=1, \iota_{k}, i, k=1, \ldots, n$, where the dual units $\iota_{k}$ are nilpotent $\iota_{k}^{2}=0$ and agree with the commutative law of multiplication $\iota_{k} \iota_{m}=\iota_{m} \iota_{k} \neq 0$. These dual values of the parameters $j$ correspond to the spaces with degenerate metrics. The main difference of affine root system is the presence of a special (imaginary) root $\delta$ with the nilpotent property $\delta^{2}=0$. Comparison of the nilpotent property of the imaginary root and the dual unit suggests that root systems in spaces with degenerated metric correspond to affine algebras. This geometrical interpretation of the root system of affine Lie algebras may help in understanding their properties.

Kac-Moody algebras are characterized by Chevalley relations

$$
\begin{gathered}
{\left[h_{i}, h_{j}\right]=0, \quad\left[h_{i}, e_{j}\right]=a_{i j} e_{j},} \\
{\left[h_{i}, f_{j}\right]=-a_{i j} f_{j}, \quad\left[e_{i}, f_{j}\right]=\delta_{i j} h_{j},}
\end{gathered}
$$


and Serre relations

$$
\left(a d e_{i}\right)^{1-a_{i j}} e_{j}=\left(a d f_{i}\right)^{1-a_{i j}} f_{j}=0
$$

where $h_{i}, e_{i}, f_{i}, i=1,2, \ldots, r$ are Weyl generators. Cartan matrix $A=\left(a_{i j}\right)$ may be given algebraically:

1) $a_{i j} \in \mathbb{Z}$,

2) $a_{i j}<0$ for $i \neq j$

3) $a_{i j} a_{j i} \leq 3$,

4) $a_{i j}=0 \Longleftrightarrow a_{j i}=0$,

5) $\operatorname{det}\left(a_{i j}\right) \neq 0$

for simple Lie algebras and 1), 2), 4), as well $\left.\left.3^{a}\right) a_{i j} a_{j i} \leq 4,5^{a}\right) \operatorname{det}\left(a_{i j}\right)=0$, the equation $\sum a_{i j} x_{j}=0$ has only one solution - for affine algebras.

All these matrices are classified and hence all simple and affine Lie algebras are listed. Affine Kac-Moody algebras was discovered by an algebraic way [8].

Cartan matrix may be also given geometrically with the help of root system. Root system of simple Lie algebra is a finite set of vectors $\tilde{\Pi}_{0}=\{\alpha, \beta, \ldots\}$ in Euclidean space $V(r)$ with a positive defined nondegenerate metric if 1) $s_{\alpha} \tilde{\Pi}_{0}=$ $\tilde{\Pi}_{0}$ for all $\left.\alpha \in \tilde{\Pi}_{0} ; 2\right)$ for all $\alpha, \beta \in \tilde{\Pi}_{0}, n(\alpha, \beta)=2 \frac{(\alpha, \beta)}{(\alpha, \alpha)} \in \mathbb{Z}$ are integers; 3) the linear span of $\tilde{\Pi}_{0}$ coincides with $V(r): \mathcal{L}\left(\tilde{\Pi}_{0}\right)=V(r)$, where

$$
s_{\alpha}(\beta)=\beta-2 \frac{(\alpha, \beta)}{(\alpha, \alpha)} \alpha
$$

is the reflection of $\beta$ with respect to $\alpha$. A subset $K \subset \tilde{\Pi}_{0}$ is called a basis $K=\left\{\alpha_{i}, i=1, \ldots, r\right\}$ of root system $\tilde{\Pi}_{0}$ if the following conditions hold: 1) $K$ form a basis of $V(r), 2) \forall \alpha \in \Pi_{0} \alpha=\sum_{i=1}^{r} m_{i} \alpha_{i}$, where $\alpha_{i} \in K, m_{i} \in \mathbb{Z}$ and all $m_{i} \geq 0$ (positive roots) or all $m_{i} \leq 0$ (negative roots). The matrix

$$
(\tilde{A})_{i j}=n\left(\alpha_{i}, \alpha_{j}\right)=2 \frac{\left(\alpha_{i}, \alpha_{j}\right)}{\left(\alpha_{i}, \alpha_{i}\right)}, \quad \alpha_{i}, \alpha_{j} \in K
$$

is called the Cartan matrix $\tilde{A}$ of root system. A set of reflections form a group $W_{0}$ called a Weyl group of root system.

Consider $(r+1)$-dimensional vector space $V(r, 1)$ equipped with a degenerate metric of signature $(0,+, \ldots,+$,$) , i.e. the scalar product of vectors \nu=$ $\left(\nu_{0}, \nu_{1}, \ldots, \nu_{r}\right)$ and $w=\left(w_{0}, w_{1}, \ldots, w_{r}\right)$ is define as $(\nu, w)=\sum_{k=1}^{r} \nu_{k} w_{k}$. According to the geometric viewpoint $V(r, 1)$ is a product bundle with $r$-dimensional Euclidean subspace $V(r)$ as a base and one-dimensional orthogonal to $V(r)$ subspace as a fiber. The projection is given by mapping $\pi: V(r, 1) \rightarrow V(r)$. At $r=3$ the space $V(3,1)$ may be interpreted as one of the possible fourdimensional space-time models, namely Carroll kinematic [10]-[12]. Therefore, we shall call $V(r, 1)$ Carroll space.

Above mentioned property of the imaginary root gives reasons for the following definitions. A vector set $\tilde{\Pi}$ in Carroll space $V(r, 1)$ is called degenerate root system (DRS), if the properties 1 )-3) of root system (with the replacement $\tilde{\Pi}_{0}$ 
by $\tilde{\Pi}, V(r)$ by $V(r, 1))$ and 4) $\forall \alpha \in \tilde{\Pi} \pi \alpha \neq 0$ are satisfied. The root system is called reduced if there are no parallel roots (the roots $\alpha$ and $-\alpha$ are antiparallel). The root system $\tilde{\Pi}$ is called reducible if $V(r, 1)=V_{1} \oplus V_{2}$ and $\tilde{\Pi}=\Pi_{1} \cup \Pi_{2}$, where $\Pi_{1}$ and $\Pi_{2}$ are the root systems in $V_{1}$ and $V_{2}$ respectively. Otherwise the root system is called irreducible. The reduced irreducible root system will be denoted by $\Pi$. The basis $B=\left\{\alpha_{i}, i=0, \ldots, r\right\}$ of $\Pi$ is defined similarly to the ordinary root system. The matrix $\bar{A}$, defined by (4) for $\alpha_{i}, \alpha_{j} \in B$ is called the Cartan matrix of DRS. It is natural to associate the vector $\varepsilon_{0}$ in $V(r, 1)$, pointing out the degeneracy direction with the imaginary root.

Proposition 1. The projection $\pi$ of degenerate root system $\Pi$ onto $V(r)$ gives the root system $\tilde{\Pi}_{0}, \pi: \Pi \rightarrow \tilde{\Pi}_{0}$.

Proof. Let us separate the vectors of DRS $\Pi=\{\alpha, \beta, \ldots\}$ into collinear and perpendicular to $\varepsilon_{0}$ components: $\alpha=\alpha_{0}+\alpha_{\perp}, \beta=\beta_{0}+\beta_{\perp}, \ldots$ From the property 4$)$ of DRS we get $\alpha_{\perp} \neq 0, \beta_{\perp} \neq 0, \ldots$ The reflections in $\Pi$ are passed into the reflections in $\tilde{\Pi}_{0}$ under the projection $\pi: s_{\alpha_{0}+\alpha_{\perp}}\left(\beta_{0}+\beta_{\perp}\right)=s_{\alpha_{\perp}}\left(\beta_{\perp}\right)$, i.e. for $\tilde{\Pi}_{0}=\left\{\alpha_{\perp}, \beta_{\perp}, \ldots\right\}$ the property 1 ) of the root system is held. Further, from $n(\alpha, \beta)=n\left(\alpha_{\perp}, \beta_{\perp}\right)$ it follows that the property 2$)$ of the root system is fulfilled. Finally, from $\mathcal{L}(\Pi)=V(r, 1)$ we obtain that the linear span of $\tilde{\Pi}_{0}$ coincides with $V(r)$, i.e. the property 3 ) is valid. Therefore $\tilde{\Pi}_{0}$ is the root system of simple Lie algebra.

Remark. An unreduced root system may be obtained at the projection of the reduced DRS. It should be noted that it will occur if DRS corresponds to the twisted affine Lie algebra $\hat{A}_{2 l}^{(2)}$.

Proposition 2. The Cartan matrix of the degenerate root system is affine.

Proof. It is enough to show that the conditions 1), 2), $3^{a}$ ), 4), $5^{a}$ ) for affine Cartan matrix follow from the definition of degenerate root system. The integerness 1) follows from the property 2) of DRS. Further, similarly to the case of an ordinary root system [13] one can show that the difference $\alpha-\beta$ is the root if $n(\alpha, \beta)>0$, i.e. the angle between the projections of vectors onto $V(r)$ is acute. For simple (basis) roots $n(\alpha, \beta)<0$, i.e. the angle between projections is obtuse therefore the condition 2 ) is held. Let $\alpha, \beta \in \Pi$ are arbitrary roots. We have the equation $n(\alpha, \beta) n(\beta, \alpha)=4 \cos \phi$, where in contrast to nondegenerate case, $\phi$ is the angle between the projections of vectors onto $V(r)$. Since the collinearity of the projections does not mean the collinearity of vectors, the case $\phi=0$ can not be rejected now, in full agreement with $3^{a}$ ). The condition 4) follows from the symmetry of scalar product. It remains to prove the conditions $5)$. It is clear that all metric properties of DRS are determined by the properties of vector projections. It was shown in Proposition 1 that these projections form the root system of simple Lie algebra. All basis roots of DRS are projected onto subspace $V(r)$, forming nonacute angles with each other. In this case one of the roots becomes linearly dependent since the number of basis roots of the root system, formed by the projections of roots of DRS, is just one less. Therefore, the condition 5) is held for the Gramm matrix, composed by the basis roots of DRS. Dividing its columns by the corresponding square lenght of basis roots, we obtain the same property for Cartan matrix. 
Degenerated root systems are called equivalent, if their Cartan matrices are the same. Since Cartan matrix of DRS is determined by its projection an equivalence class is formed by all DRS with the same projections onto $V(r)$. In general case the projection $\Pi$ onto $V(r)$ is not contained in $\Pi$. $\Pi$ is called canonical DRS if $\pi \Pi \backslash P=\Pi_{0} \subset \Pi$, where $P=\{\beta \in \pi \Pi \mid 2 \beta \in \pi \Pi\}$ or $P=\left\{\beta \in \pi \Pi \mid \frac{1}{2} \beta \in \pi \Pi\right\}$, i.e. if reduced root system $\Pi_{0}$ is contained in subspace $V(r)$.

Let us construct the basis $B=\left\{\alpha_{0}, K\right\}=\left\{\alpha_{0}, \alpha_{1}, \ldots, \alpha_{r}\right\}$ of canonical DRS. It is clear that as $K$ should be taken the basis of root system $\Pi_{0}$. It remains to construct the $(r+1)$-th basis root $\alpha_{0}=\pi \alpha_{0}+\delta \epsilon_{0} \in V(r, 1), \delta \in R$, that is in fact to choose the projection $\pi \alpha_{0}$ in subspace $V(r)$ in such a way that the set of vectors $\left\{\pi \alpha_{0}, K\right\}$ generate according to the Proposition 2 affine Cartan matrix. It is clear that $\pi \alpha_{0}$ is such a linear combination of vectors from $K$, that $\left(\pi \alpha_{0}, \alpha_{k}\right) \leq 0, k=1, \ldots, r$. As it was mentioned in Proposition $1 \tilde{\Pi}_{0}=\pi \Pi$ may be both reduced and irreducible root system.

Let $\tilde{\Pi}_{0}=\Pi_{0}$ be a reduced root system, then $\pi \alpha_{0} \in \Pi_{0}$. When roots of different length are presented in $K=\left\{\alpha_{1}, \ldots, \alpha_{r}\right\}$, two cases are possible.

1) $\left|\pi \alpha_{0}\right|=\left|\alpha_{l}\right|$. All root systems $\Pi_{0}$ of simple Lie algebras have the highest vector $\theta=\sum_{k=1}^{r} a_{k} \alpha_{k}$, where $a_{k}$ are the marks on Dynkin diagramms of simple Lie algebras. The properties $\left(\theta, \alpha_{k}\right) \leq 0, k=1, \ldots, r$, are satisfied for this highest root and also $|\theta|=\left|\alpha_{l}\right|$. (When all roots in $K$ have the same length, we consider them as long roots). By setting $\pi \alpha_{0}=-\theta$ we obtain the bases $B$ of root systems of all untwisted affine algebras [8].

2) $\left|\pi \alpha_{0}\right|=\left|\alpha_{s}\right|$. Root systems $\Pi_{0}$ of simple Lie algebras $C_{r}, B_{r}, F_{4}, G_{2}$ contain, except $\theta$, the root $\tilde{\theta}=\sum_{k=1}^{r} \tilde{a}_{k} \alpha_{k}$, where $\tilde{a}_{k}$ are Kac marks on Dynkin diagramm of affine algebras. The properties $\left(\tilde{\theta}, \alpha_{k}\right) \leq 0, k=1, \ldots, r$, are satisfied and $|\tilde{\theta}|=\left|\alpha_{s}\right|$. We obtain the bases $B$ of root systems of all twisted affine Lie algebras, except $\hat{A}_{2 r}^{(2)}$, by setting $\pi \alpha_{0}=-\tilde{\theta}[8]$.

Let now $\tilde{\Pi}_{0}=\Pi_{0} \cup P$ be a nonreduced root system (there are collinear vectors with the length twice as little as the length of long roots $\alpha_{l} \in \Pi_{0}$ or with the length twice as much as the length of short roots $\left.\alpha_{s} \in \Pi_{0}\right)$. If $\pi \alpha_{0} \in \Pi_{0}$, then it follows from above consideration, that $\pi \Pi=\Pi_{0}$ is a reduced root system. But in our case $\tilde{\Pi}_{0}$ is nonreduced, therefore the required DRS can not be constructed in this way. It remains to regard the case $\pi \alpha_{0} \notin \Pi_{0}$. When $\left|\pi \alpha_{0}\right|=\frac{1}{2}\left|\alpha_{l}\right|$, then $\pi \alpha_{0}=-\frac{1}{2} \theta$ and when $\left|\pi \alpha_{0}\right|=2\left|\alpha_{l}\right|$, then $\pi \alpha_{0}=-2 \theta$, where $\theta$ is the highest root of $\Pi_{0}$. In this case we obtain the bases $B$ of root systems of twisted affine algebras $\hat{A}_{2 r}^{(2)}[8]$.

Thus, we have constructed the bases of root systems of all affine Lie algebras, relying on the properties of root systems of simple Lie algebras and on the properties of DRS.

Reflections of DRS form Weyl group $W$. This group is generated by the basic reflections $s_{\alpha_{0}}, s_{\alpha_{1}}, \ldots, s_{\alpha_{r}}$. It is obvious that last $r$ basic reflections generate the subgroup $W_{0}$. Consider the $k$-th power of translation operator of root $x$ 
along $\varepsilon_{0}$, which is constructed from reflections

$$
t_{\alpha}^{k}(x) \stackrel{d f}{=} s_{k \delta \varepsilon_{0}-\alpha} s_{\alpha}(x)=x-2 \frac{(\alpha, x)}{(\alpha, \alpha)} k \delta \varepsilon_{0},
$$

where $\alpha, k \delta \varepsilon_{0}-\alpha, x \in \Pi,(\alpha, x) \neq 0$. The set of translations generates a commutative translation subgroup $T$. It is easily to show that Weyl group $W$ is a semidirect product of its subgroup $W=W_{0} \triangleright T$.

Now we determine the structure of DRS. It is enough to take into account the fact, that the root length and its component $\varepsilon_{0}$ are not changed under the reflections: $w\left(\delta \varepsilon_{0}\right)=\delta \varepsilon_{0}, w \in W_{0}$, i.e. subgroup $W_{0}$ acts transitively on the set of equal length roots with the same $\varepsilon_{0}$ coordinate and to construct the shifts operators for different length roots. The complete root system is the join of imaginary and real roots: $\Pi=\Pi_{r e} \cup \Pi_{i m}$, where $\Pi_{i m}=\left\{n \delta \varepsilon_{0} \mid n \in Z\right\}$. It remains to build $\Pi_{r e}$.

Consider first $\Pi_{r e}$ of untwisted affine Lie algebras. The root $\alpha_{0}$ has maximal length $\left|\alpha_{0}\right|=\left|\alpha_{l}\right|$ for this algebras. The subset of shifted long roots $\left\{\Pi_{0}^{l}+\delta \varepsilon_{0}\right\}$ is obtained by acting reflections from $W_{0}$ on $\alpha_{0}=\pi \alpha_{0}+\delta \varepsilon_{0}, \pi \alpha_{0} \in \Pi_{0}$. The short root $\alpha_{m}$ is shifted by operator $t_{\alpha_{m-1}}\left(\alpha_{m}\right)=\alpha_{m}+\delta \varepsilon_{0}$, where $\alpha_{m-1}$ is a long root, connected to $\alpha_{m}$ on Dynkin diagramm. Here $m=1$ for $C_{r}^{(1)}, m=2$ for $G_{2}^{(1)}, m=3$ for $F_{4}^{(1)}, m=r$ for $B_{r}^{(1)}$. Using reflections from $W_{0}$ we obtain a set $\Pi_{0}^{s}+\delta \varepsilon_{0}$ of shifted short roots. As far as $\Pi_{0}^{l} \cup \Pi_{0}^{s}=\Pi_{0}$ for untwisted algebras, then $\Pi_{0} \subset V(r)$ pass to $\Pi_{0}+\delta \varepsilon_{0} \subset V(r, 1)$. The next step is the shift of long root $\alpha_{0}: t_{\alpha_{p}}\left(\alpha_{0}\right)=\alpha_{0}+\delta \varepsilon_{0}$, where $p=6$ for $E_{6}^{(1)}, p=2$ for $B_{r}^{(1)}, D_{r}^{(1)}, p=1$ for remaining untwisted affine algebras. The repetition of shift procedure of long and short roots leads to the set $\left\{\Pi_{0}+2 \delta \varepsilon_{0}\right\}$. The shift of the set $\Pi_{0}$ in negative direction of axis $\varepsilon_{0}$ is constructed in a similar way. Thus, DRS of untwisted affine Lie algebras is as follows

$$
\Pi_{r e}=\left\{\Pi_{0}+n \delta \varepsilon_{0} \mid n \in Z\right\} .
$$

For twisted algebras (except of $A_{2 r}^{2}$ ) the root $\alpha_{0}$ has minimal length $\left|\alpha_{0}\right|=$ $\left|\alpha_{s}\right|$. The subset of short shifted roots $\left\{\Pi_{0}^{s}+\delta \varepsilon_{0}\right\}$ is obtained by acting of reflections from $W_{0}$ on $\alpha_{0}=\pi \alpha_{0}+\delta \varepsilon_{0}, \pi \alpha_{0} \in \Pi_{0}$. The long root $\alpha_{m}$ is shifted by operator $t_{\alpha_{m-1}}\left(\alpha_{m}\right)=\alpha_{m}+2 \delta \varepsilon_{0}$, where $\alpha_{m-1}$ is a short nonorthogonal to $\alpha_{m}$ root. Here $m=1$ for $D_{r+1}^{(2)}, m=2$ for $D_{4}^{(3)}, m=3$ for $E_{6}^{(2)}, m=r$ for $A_{2 r-1}^{(2)}$. The set $\left\{\Pi_{0}^{l}+2 \delta \varepsilon_{0}\right\}$ is obtained with the help of reflections from $W_{0}$. The next step is the shift of short roots. The root $\alpha_{0}$ is shifted by $t_{\alpha_{p}}\left(\alpha_{0}\right)=$ $\alpha_{0}+\delta \varepsilon_{0}=\pi \alpha_{0}+2 \delta \varepsilon_{0}, p=1$ for $D_{4}^{(3)}, E_{6}^{(2)}, p=2$ for $A_{2 r-1}^{(2)}$ and the root $\alpha_{r}$ is shifted by $t_{\alpha_{r-1}}^{2}\left(\alpha_{r}\right)=\alpha_{r}+2 \delta \varepsilon_{0}$ in the case of $D_{2 r-1}^{(2)}$ algebra. (The operator $t_{\alpha_{r-1}}=s_{\delta \varepsilon_{0}-\alpha_{r-1}} s_{\alpha_{r-1}}$ is not defined now because $\delta \varepsilon_{0}-\alpha_{r-1} \notin \Pi$, therefore we have use the operator $\left.t_{\alpha_{r-1}}^{2}\right)$. The shifted short roots are transformed by subgroup $W_{0}$ to the set $\left\{\Pi_{0}^{s}+2 \delta \varepsilon_{0}\right\}$. The set $\left\{\Pi_{0}^{l}+2 \delta \varepsilon_{0}\right\}$ of shifted long roots is transformed by $W_{0}$ and by the operator $t_{\alpha_{m-1}}^{2}\left(\alpha_{m}\right)=\alpha_{m}+4 \delta \varepsilon_{0}$ to the set $\left\{\Pi_{0}^{l}+4 \delta \varepsilon_{0}\right\} . \Pi_{r e}$ of twisted affine algebras (except of $A_{2 r}^{(2)}$ ) is the join of the 
sets of long and short shifted roots.

$$
\Pi_{r e}=\left\{\Pi_{0}^{s}+n \delta \varepsilon_{0}\right\} \cup\left\{\Pi_{0}^{l}+n k \delta \varepsilon_{0}\right\},
$$

$n \in Z, k=2,3$.

Now we find the construction of $\Pi_{r e}$ for algebras $A_{2 r}^{(2)}$, where $\left|\alpha_{0}\right|=\frac{1}{2}\left|\alpha_{l}\right|<$ $\left|\alpha_{s}\right|<\left|\alpha_{l}\right|$ and $\pi \alpha_{0} \notin \Pi_{0}$. The operator $t_{\alpha_{0}}$ shifts the short root $\alpha_{1} \in \Pi_{0},\left|\alpha_{1}\right|=$ $\left|\alpha_{s}\right|$ on $2 \delta \varepsilon_{0}: t_{\alpha_{0}}\left(\alpha_{1}\right)=\alpha_{1}+2 \delta \varepsilon_{0}$. The operator $t_{\alpha_{1}}=s_{\left(\delta \varepsilon_{0}-\alpha_{1}\right)} s_{\alpha_{1}}$ is not defined since $\delta \varepsilon_{0}-\alpha_{1} \notin \Pi$, therefore the shift operators of short roots $\alpha_{k}$ are as follows: $t_{\alpha_{k}}^{2}\left(\alpha_{k+1}\right)=\alpha_{k+1}+2 \delta \varepsilon_{0}, k=1,2, \ldots, r-2$. The shifted short roots are transformed by $W_{0}$ to the set $\Pi_{0}^{s}+2 \delta \varepsilon_{0}$. The short roots are shifted with the help of operators $t_{\alpha_{0}}^{n}, t_{\alpha_{k}}^{n}, n \in Z$ on $2 n \delta \varepsilon_{0}$ and then are reproduced by $W_{0}$. As a result we obtain the subset $\Pi_{1}=\left\{\Pi_{0}^{s}+2 n \delta \varepsilon_{0} \mid n \in Z\right\}$. The long root $\alpha_{r} \in \Pi_{0},\left|\alpha_{r}\right|=\left|\alpha_{l}\right|$ is shifted on $4 \delta \varepsilon_{0}: t_{\alpha_{r-1}}^{2}\left(\alpha_{r}\right)=\alpha_{r}+4 \delta \varepsilon_{0}$. Similarly, using the shift operators $t_{\alpha_{r-1}}^{2 n}$ and reflections from $W_{0}$, we obtain the subset of long roots $\Pi_{2}=\left\{\Pi_{0}^{l}+4 n \delta \varepsilon_{0} \mid n \in Z\right\}$.

It remains to explain the construction of roots of length $\left|\alpha_{0}\right|=\frac{1}{2}\left|\alpha_{l}\right|$. The reflections from $W_{0}$ transform the basis root $\alpha_{0}=\pi \alpha_{0}+\delta \varepsilon_{0}$ to the set of roots $\left\{\frac{1}{2} \Pi_{0}^{l}+\delta \varepsilon_{0}\right\}$, since $\pi \alpha_{0}=-\frac{1}{2} \theta$. Operator $t_{\alpha_{1}}^{2}$ shift $\alpha_{0}$ on $2 \delta \varepsilon_{0}: t_{\alpha_{1}}^{2}\left(\alpha_{0}\right)=$ $\alpha_{0}+2 \delta \varepsilon_{0}=\pi \alpha_{0}+3 \delta \varepsilon_{0}$ and then this shifted root is reproduced by the reflections from subgroup $W_{0}$ to the set $\left\{\frac{1}{2} \Pi_{0}^{l}+3 \delta \varepsilon_{0}\right\}$. Operators $t_{\alpha_{1}}^{2 n}, n \in Z$ and reflections from $W_{0}$ generate the subset $\Pi_{3}=\left\{\frac{1}{2} \Pi_{0}^{l}+(2 n+1) \delta \varepsilon_{0} \mid n \in Z\right\}$. Then the set of real roots of $A_{2 r}^{(2)}$ is the join of the subsets

$$
\begin{aligned}
\Pi_{r e}=\left\{\frac{1}{2} \Pi_{0}^{l}+(2 n+1) \delta \varepsilon_{0}\right\} & \cup\left\{\Pi_{0}^{s}+2 n \delta \varepsilon_{0}\right\} \cup \\
& \left\{\Pi_{0}^{l}+4 n \delta \varepsilon_{0}\right\}, n \in Z .
\end{aligned}
$$

Thus, with the help of geometric consideration using the shifts operators of basic roots along $\varepsilon_{0}$ and the reflections from subgroup $W_{0}$ we have obtained sufficiently easily the structure of DRS in Carroll spaces. It is natural that our results are coincided with the analogous ones for affine root systems in pure algebraic consideration.

The analysis of other types of Kac-Moody algebras which are discussed in literature shows that their Cartan matrices may be obtained with the help of geometric consideration. For example, root systems in pseudoeuclidean spaces are connected with hyperbolic Kac-Moody algebras [14] and Borcherds algebras [15],[16]. In this case the algebraic condition $a_{i i}=0$ on symmetrized Cartan matrix corresponds geometrically to the case, when the basic root belongs to the con $(x, x)=0$ and the condition $a_{i i}<0$ means, that basis roots have both positive and negative length, i.e. they are situated in different con sectors.

The root systems in the space with twice degenerate metric are connected apparently with toroidal algebras [17],[18]. This geometric interpretation makes possible to establish some properties of their Cartan matrices. So the condition $5^{a}$ ) is changed and the property $a_{i j}<0$ at $i \neq j$ is not more held since it is impossible to distribute $n+2$ projections of basis roots on $n$-dimensional 
Euclidean space so that all angles between these roots are nonacute, i.e. all scalar products between them are nonpositive. It is clear that this imply the modification of Serre relations.

A generalization of the observation indicated in the letter leads to the suggestion: to regard root systems in spaces of constant curvature (especially with degenerate metric) and to construct corresponding Lie algebras. The work on this problem is continued.

\section{References}

[1] N.A.Gromov, Int. J. of Theor. Phys. 29 (1990) 607-620.

[2] E.Inönü, E.P.Wigner, Proc.Nat.Acad.Sci. USA 39 (1953) 510.

[3] M. de Montigny, J.Patera, 1991 J.Phys.A: Math.Gen. 24 (1991) 525, R.V.Moody, J.Patera, J.Phys.A: Math.Gen. 24 (1991) 2227.

[4] C.Nappi and E.Witten, Phys. lett. B293 (1992) 309.

[5] D.Olive, E.Rabinovici and A. Schwimmer, Phys. lett. B321 (1994) 361.

[6] A.Aghamohammadi, M.Khorrami, A.Shariati, hep-th/9810001

[7] N.A.Gromov, I.V.Kostyakov and V.V.Kuratov, Int. J. of Mod. Phys. 12 (1997) 183-188

[8] V. Kac, Infinite Dimensional Lie Algebras (1985) (Cambridge: Cambridge University Press)

[9] R.I. Pimenov, Litovskii matemat. sbornik 5 (1965) 457-486 (in Russian).

[10] N.A. Gromov, Contractions and Analytical Continuation of Classical Groups. Unified Approach (Syktyvkar: Komi Science Center) (1990) (in Russian).

[11] H. Bacry and J-M. Levy-Leblond, J. Math. Phys. 9 (1968) 1605-1614.

[12] J-M. Levy-Leblond, Ann. Inst. H.Poincare A3 (1965) 1-12.

[13] J-P. Serre, Lie Algebras and Lie Groups (1965) (New-Amsterdam: Benjamin)

[14] V.V. Nikulin, alg-geom/9810001, 9711032, 9712033, 9905150.

[15] R.E. Borcherds, J. of Algebra 115 (1988) 501-512.

[16] R.M. Gebert, hep-th/9308151.

[17] Rao S. Eswara and R.V. Moody, Commun. Math. Phys. 159 (1994) 239264.

[18] Inami Takeo, Kanno Hiroaki and Ueno Tatsuya, hep-th/9610187. 